\title{
Laparoscopic treatment of hepatic hydatid disease: Şanlıurfa-Turkey experience and early clinical outcomes
}

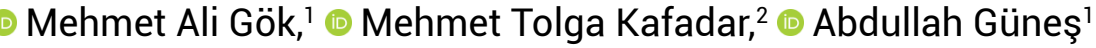 \\ 'Department of General Surgery, Health Sciences University Derince Training and Research Hospital, Kocaeli, Turkey \\ 2Department of General Surgery, Health Sciences University Mehmet Akif İnan Training and Research Hospital, Şanlıurfa, Turkey
}

\begin{abstract}
Introduction: Cyst hydatid disease is still our territory and remains a common health problem for our country. In this clinical retrospective study, cases of liver hydatid cysts treated as laparoscopic were analysed.

Materials and Methods: Medical records of 25 patients ( 16 female, 9 male; mean age $41 \pm 3.42$; range 20-78) who were treated for hepatic hydatid disease in our clinic between June 2016 and June 2017 were retrospectively reviewed. Patients were evaluated in terms of age, sex, location of cyst, surgical method applied, duration of hospital stay, postoperative complications and treatment methods for complications.

Results: One patient (4\%) due to intraperitoneal rupture was operated urgently. In $5(20 \%)$ of the cases, bile leakage were seen in the cyst sponge during the operation and perioperative laparoscopic suture was performed. In three of these patients (12\%), the leakage was closed without any intervention, two ( $8 \%$ ) lasted more than a week and the daily flow was over $150 \mathrm{cc}$. Endoscopic sphincterotomy was applied to these cases.

Conclusion: Nowadays at experienced centers, laparoscopic surgical interventions in selected patients take place in the treatment algorithm as an effective and safe method for the treatment of liver cyst hydatid disease. We think that uncomplicated hydatid cysts do not need specialized laparoscopic instruments in the surgery.
\end{abstract}

Keywords: Hydatid cyst; laparoscopic surgery; liver.

\section{Introduction}

Echinococcosis which is caused mostly by Echinococcus granulosus and rarely by Echinococcus multilocularis is a parasitic and zoonotic disease seen in Eastern and South eastern Anatolia of our country where the common source of livelihood is animal husbandry. Eating vegetables contaminated with stools of canidae which are defin- itive hosts and carry the eggs, the envelopes of the eggs are opened in the stomach of intermediate hosts such as sheep and cattle. Passing the mucosa in the intestine,released larva reach to river with portal blood flow. Unable to pass through the liver sinusoids, they may cling to the lungs and other peripheral organs. In the inner part of the host's tissue, they form a germinative layer (endocyst) re- 
sponsible for the construction of hydatic fluid and development of scolex and small cysts. On the other hand, in the outer part they form cystic structures that have a layer (ectocyst) with opaque laminae. This growing cystic structure is externally confined to a fibrous adventitial capsule (pericyste), which is formed by inflammatory cells and fibroblasts of the host's tissue. Although the causative agent may find harbor in almost all tissues of humans that are accidental intermediate hosts, the most common organ is the liver. ${ }^{[1]}$ Patients are most of the times asymptomatic; thus, the size of the cysts may develop causing specific symptoms or result in a dramatic situation due to spreading up to the intrahepatic biliary tract. Although a good history and physical examination constitute the basis for clinical diagnosis, it should be supported by ultrasonography (USG) and computed tomography (CT). Even though serological methods such as "Enzyme-Linked Immunosorbent Assay" (ELISA) test, "indirect hemagglutination" test and "indirect fluorescent antibody" tests are used, and the fact that tests proved negative do not allow a precise exclusion from the hydatid cyst diagnosis. Invasive procedures such as percutaneous aspiration, laparoscopic and open cystectomy, unroofing and radical resection are utilized in the treatment together with antiparasitic agents like albendazole. The use of minimally invasive methods for hepatic surgery is becoming more widespread, largely due to increased experience in laparoscopic surgery and availability of new technologies. Indeed, several reports comparing laparoscopic to open liver surgery have reported a significant decrease of intraoperative blood loss, reduced postoperative stay, and lower complication rate, while maintaining safety and effectiveness. The published clinical experiences employing minimally invasive approaches for radical treatment of liver hydatid disease yielded excellent outcomes. Indeed, both laparoscopy and robotics can be performed safely with a low rate of complications while permitting an effective and radical therapy ${ }^{[2]}$ In this article, cases with liver hydatid cyst which we treated by laparoscopy are presented.

\section{Materials and Methods}

A total of 25 patients with liver hydatic cysts applied to Health Sciences University Mehmet Akif İnan Training and Research Hospital Clinic of General Surgery between June 2016 and June 2017 with having evaluations of Type I, Type II and Type III according to the Gharbi classification in USG and whom were not planned percutaneous aspiration due to technical and social reasons had laparoscopic cystectomy + unroofing surgery without using specific devices. After anamnesis and physical examination, whole blood count and biochemical data were evaluated. Serologic testing (indirect hemagglutination test) was applied to each patient. In the preoperative period, abdominal ultrasonography and computed tomography were performed for detailed investigation of the location and composition of the cystic structure. Each patient was treated with albendazole (15 mg/kg/day) as a 3 week intravascular intervention for one week prior to operation and 3 months postoperatively. This treatment was not possible for perforated cyst, because it was operated urgently. All 25 patients included in the study had laparoscopic operation. Two patients had upper abdominal surgical history. One of the patients had an emergency operation due to cyst rupture. Two patients had a record with hydatid cyst surgery. After endotracheal general anesthesia, the patients were taken to the supine position. The imaging system was placed in the area where the patient's right shoulder was located. After mini-incision under umbilicus, a pneumoperitoneum was created to provide intraabdominal pressure of $14 \mathrm{mmHg}$ with 10 $\mathrm{mm}$ trocars placed under the direct eyesight. A 30-degree laparoscopic camera inserted through a $10 \mathrm{~mm}$ trocar to image inside the abdomen. One piece of $5 \mathrm{~mm}$ and another piece of $10 \mathrm{~mm}$ trocars were placed according to the location of the lesion. A 20\% serum saline buffer was placed around the cyst. With a veress needle inserted directly into the cyst from a separate point, the cyst contents were first drained, then $20 \%$ serum saline was injected and waited for 10 minutes and the pressure inside the cyst was lowered by aspiration again. Opening the cystic wall with cautery and scissors, the liquid content was aspirated. Then the cyst wall was excised and the entire germinative and laminar membrane were enabled to release from the cavity and removed from the abdomen with the help of endobag. Skolocidal agent impregnated buffers were taken out of the abdomen by means of endobag. Five patients were observed to have biliary leakage in the cyst cavity during surgery. The cases were laparoscopically sutured (Fig. 1). Then the operation was finalized by placing an aspirative drain into the cavity. All patients were treated by the same surgical team with the same standard technique.

\section{Statistical Analysis}

For the statistical analysis, SPSS computer program (Statistical Package for the Social Sciences, version 21.0, SPSS Inc., Chicago, Illinios, USA) was used. Since the number 
of samples $<50$, the normalization of the distribution was examined by Shapiro-Wilktest. Descriptive statistics were expressed as mean \pm standard deviation values for data in compliance with normal distribution and as median and minimum-maximum values for data not in compliance with the normal distribution. The relationship between values in accordance with the normal distribution was assessed with Pearson correlation test and the relationship between the values fail to comply with normal distributions was assessedby the Spearman correlation test and the results $\mathrm{p}<0.05$ were considered to be significant in terms of correlation.

\section{Results}

One of the patients (4\%) applied to the emergency room with severe abdominal pain. Of the twentyfour patients (96\%), the diagnosis was made incidentally due to imaging methods performed at the outpatient clinics with various complaints. The echinococcal cysts in all patients were solitary cysts with liver localization. Sixteen of the patients are female whereas 9 were male. The mean age of the patients was $41 \pm 3.42$. The youngest patient was 20 years old, the oldest patient was 78 years old. Ultrasonographically, the longest mean diameter was determined as $92.6 \pm 8.53 \mathrm{~mm}$ (min: $50 \mathrm{~mm}$; max: $130 \mathrm{~mm}$ ). When the anatomical location of the cysts in the liver was examined, it was seen that thirteen patients had segment 8 , six patients had segment 4, two patients segment 6, two patients segment 7 , and the other patients respectively had segment 2 and segment 5 located cysts. Indirect haemagglutination test was negative (1/32) in three of the twentyfive patients (12\%) and positive (1/128) in the other patients. In negative patients, the diagnosis of hydatid cyst was confirmed pathologically in the postoperative period with the presence of germinative membrane. Preoperatively, eight patients (32\%) were assessed as American Society of Anesthesiologists (ASA) 1, twelve patients (48\%) as ASA 2, and five patients (20\%) as ASA 3. All patients underwent laparoscopic unroofing + total cystectomy + drainage. All operations were performed with standard laparoscopic hand tools. No open surgery was performed in any patient. Mean operative time was $58 \pm 6.91$ minutes. The longest operation duration was 115 minutes and the shortest operation duration was 35 minutes. No perioperative bleeding occurred in any of the patients. Five patients were found to have bile duct involvement during the operation. The diameter of the bile related cysts was the largest $12 \mathrm{~cm}$, smallest $7 \mathrm{~cm}$. Primary closure was performed laparoscopically. In two patient bile fluid was observed from the postoperative drain (daily $150 \mathrm{cc}$ ). Post-

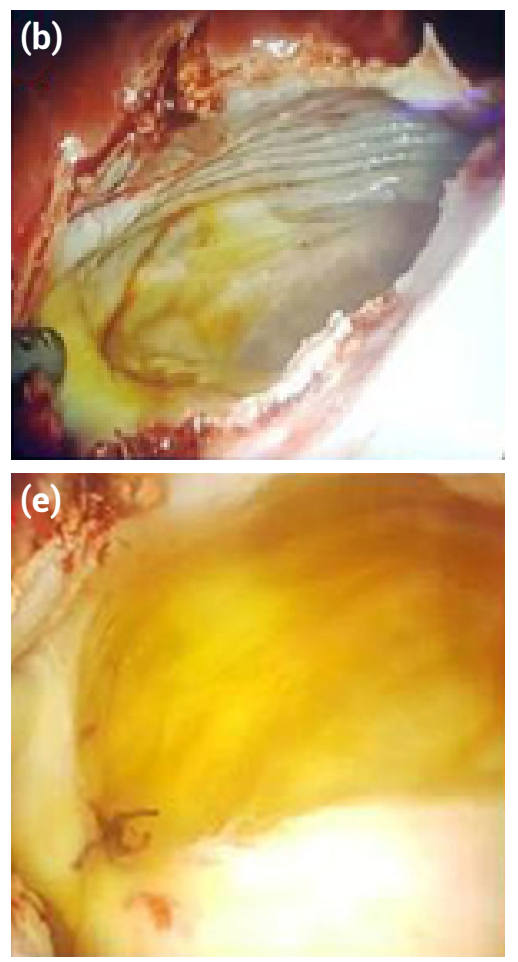


Figure 1. Intraoperative appearance of the hydatid cyst in the liver; view of the daughter vesicle (a), relation of the cyst with bile duct (b), laparoscopic suturation of the bile duct (c-e), view of the hydatid cyst adherent to diafragm (f). 
operative Endoscopic retrograde cholangiopancreatography (ERCP) was applied to these two cases. During the patients follow-up, bile drainage was reduced and the bile fistula closed spontaneously. The mean duration of hospitalization was 3 days (min: 2; max: 9). The patients developed biliary fistula were discharged on the 8 th and 9 th postoperative days after ERCP. There was no mortality in our study. Wound infection complication was seen in 1 patient. Mean duration of patient follow-up was $8 \pm 2.15$ months. After surgery all patients were followed- up with abdominal USG. Only one of the patients who were followed-up at the outpatient clinic and evaluated with USG every 3 months, recurrence was diagnosed at the postoperative 6 th month. The size of the cyst can increase as the duration of the disease increases with age. There was no correlation between the age of the patients and the diameter of the cyst in our study ( $\mathrm{p}=0.360)$. There was no significant correlation between age and operation duration $(p=0.417)$. The relationship between diameter of the cyst and operation duration was found statistically significant $(\mathrm{p}=0.005)$. It was identified that as the cystic diameter increased, the operation duration extended.

\section{Discussion}

With regards to the last two decades, surgical interventions have started to turn towards the minimal invasive approach from conventional methods in the treatment of liver hydatid cyst diseases. Minimal invasive techniques such as percutaneous drainage procedures and laparoscopic approaches have started to replace the classical surgical approaches. ${ }^{[3,4]}$ Laparoscopic treatment was first described in 1992, and in recent years there has been a steady growth especially in the laparoscopic approach. The technique chosen is either PAIR (percutaneous, aspiration, injection and re-aspiration) or laparoscopic surgery; the main purpose of the treatment is to prevent cystic inactivation and spread of cystic content into the abdomen like the conventional open cyst hydatid surgery in addition to the clearance of the cystic content and minimization of the cyst cavity. In the percutaneous aspiration method, the fluid in the cyst is aspirated in the presence of USG or CT and a scolosidal substance is introduced into the cavity, followed by re-aspiration. ${ }^{[5]}$

In laparoscopic surgical interventions, less postoperative pain develops compared to conventional open surgical procedures, patients are mobilized earlier and require less blood transfusions. In addition, postoperative adhesion development is significantly less in laparoscopic surgery.
After laparoscopic surgery, the duration of hospital stay and return to work is shortened, and the incidence of wound infection and incisional hernia decrease. ${ }^{[6-8]} \mathrm{La}-$ paroscopic interventions have been widely discussed in various publications in the literature because the high intraperitoneal pressure due to pneumoperitoneum causes the cystic content to spread into the abdomen and results in the possibility of anaphylactic shock risk. Surgeons' experience has improved over time, and many publications have reported that there is little or no intraoperative spread in many studies. ${ }^{[9]}$ In all patients undergoing laparoscopic surgery, we placed $20 \%$ serum saline impregnated buffers around the cyst. We then aspirated the fluid in the cyst. Therefore, we tried to minimize the risk of spreading. To prevent abdominal contamination, we kept the gauze around the cyst which were placed for purpose of barrier until the end of the operation. We did not encounter anaphylaxis in any of the patients.

Laparoscopically partial or total pericystectomy and even radical resection are also preferred in appropriate patients. In our study, all patients received cystectomy where laparoscopically germinative and the laminar membrane was completely excised. Although it is known that pericyste does not contain scolex, many studies have suggested that pericystectomy reduces recurrence, and many authors suggest this technique, which is relatively more radical than cystectomy and pouch reducing surgeries. ${ }^{[10-12]}$ In a study conducted by Tai et al., ${ }^{[13]}$ it was stated that the average operation duration of pericystectomy was 24 minutes longer than that of the cystectomy and there were no bleeding in 17 cystectomy series. It was also pointed out that in pericystectomy series, there was an average of $110 \mathrm{ml}$ bleeding and patient's stay in hospital with laparoscopic pericystectomy is extended 1 more day compared to cystectomy . The mean duration of surgery in our study was 65 minutes. We did not use specialized laparoscopic hand tools in our patients. When we evaluated the data of the patients in the series, we found a significant correlation between the duration of the operation and the diameter of the cyst. The average hospital stay in the hospital was 3 days, which was consistent with the literature for laparoscopic cystectomy. None of the patients had any bleeding during or after the operation, which was consistent with the rates in the literature. ${ }^{[14]}$ With reference to the comprehensive literature published on laparoscopic hydatid cyst surgery, Tuxun et al. ${ }^{[15]}$ point out a recurrence rate of $0-11 \%$ in laparoscopic cystectomy, which is still the most preferred method with $75.16 \%$. In our study, there 
was recurrence in one of the patients at the end of a mean follow-up period of 8 months. The patient underwent conventional surgery at another center with own request.

The rate of morbidity in laparoscopic hydatid cyst surgery is $15.1 \%$ in literature. ${ }^{[16]}$ Cystic expansion to the intrabiliary system can be seen especially in large sized cysts. When we detect cyst-related bile duct, we are able to ligate with perioperative laparoscopic suturation. When there is biliary leakage observed in the postoperative period, we decide in accordance with the clinic of the patient and daily flow rate of the drain. In patients without decreased flow rates of the drain, the pressure of the bile ducts may be reduced with sphincterotomy during ERCP. The rate of biliary leakage in the literature is reported as $6.24 \%$. ${ }^{[17]}$ In our study, perioperative biliary leakage was seen in five $(20 \%)$ patients. In these patients, it was ligated with laparoscopic suture during the surgery. In three of the patients, the leak closed without requiring any procedure. ERCP was performed in two patients due to biliary leakage in the postoperative period. After sphincterotomy, there was a decrease in the flow rate, and the patients were discharged with cure on the $8^{\text {th }}$ and $9^{\text {th }}$ days.

\section{Conclusion}

Many issues where laparoscopic surgery is superior to conventional open surgery such as morbidity, postoperative pain, wound infection, wound healing, time spent in hospital, return to daily life, incision scar, etc., are also applicable advantages in laparoscopic hydatid cyst surgery, as well. Laparoscopic hydatid cyst surgery is a surgical technique that combines open surgery with the benefits of percutaneous interventions. We think that removing the endocyst and exocyste that we routinely extract is sufficient. We believe that laparoscopic cystectomy is sufficient for this method, which is technically easier and faster, where the removal of pericyste, a fibrous layer without a scolex, is not meaningful for recurrence.

\section{Disclosures}

Ethichs Committee Approval: Retrospective study.

Peer-review: Externally peer-reviewed.

Conflict of Interest: None declared.

\section{References}

1. Shrestha SK, Thapa PB, Maharjan DK, Tamang TY. Laparoscopic Approach for Management of Hydatid Cyst of Liver. J
Nepal Health Res Counc 2017;15:67-70. [CrossRef]

2. Chen X, Cen C, Xie H, Zhou L, Wen H, Zheng S. The Comparison of 2 New Promising Weapons for the Treatment of Hydatid Cyst Disease: PAIR and Laparoscopic Therapy. Surg Laparosc Endosc Percutan Tech 2015;25:358-62. [CrossRef]

3. Duta C, Pantea S, Lazar C, Salim A, Barjica D. Minimally Invasive Treatment of Liver Hydatidosis. JSLS. 2016;20(1).

4. Zou H, Luo L, Xue H, Wang G, Wang X, Luo L, et al. Preliminary experience in laparoscopic resection of hepatic hydatidectocyst with the Da Vinci Surgical System (DVSS): a case report. BMC Surg. 2017;17:98. [CrossRef]

5. Giorgio A, Calisti G, de Stefano G, Farella N, Scognamiglio U, Giorgio V. Percutaneous treatment of hydatid liver cysts: an update. Recent Pat Antiinfect Drug Discov 2012;7:231-6.

6. Koea JB. Laparoscopic treatment of hepatic hydatid disease. ANZ J Surg 2012;82:499-504. [CrossRef]

7. Polat FR. Hydatid cyst: open or laparoscopic approach? A retrospective analysis. Surg Laparosc Endosc Percutan Tech 2012;22:264-6. [CrossRef]

8. Nari GA, Palacios Rodriguez Ó, Russo N, Figueras J. Laparoscopic approach to liver hydatidosis: initial experience. Cir Esp 2015;93:248-51. [CrossRef]

9. Zaharie F, Bartos D, Mocan L, Zaharie R, lancu C, Tomus C. Open or laparoscopic treatment for hydatid disease of the liver? A 10-year single-institution experience. Surg Endosc 2013;27:2110-6. [CrossRef]

10. Lv H, Jiang Y, Peng $X$, Zhang S, Wu X, Yang H, et al. Echinococcus of the liver treated with laparoscopic subadventitial pericystectomy. Surg Laparosc Endosc Percutan Tech 2013;23:e49-53. [CrossRef]

11. Li H, Shao Y, Aji T, Zhang J, Kashif K, Ma Q, et al. Laparoscopic approach for total cystectomy in treating hepatic cystic echinococcosis. Parasite 2014;21:65. [CrossRef]

12. Misra MC, Khan RN, Bansal VK, Jindal V, Kumar S, Noba AL, et al. Laparoscopic pericystectomy for hydatid cyst of the liver. Surg Laparosc Endosc Percutan Tech 2010;20:24-6. [CrossRef]

13. Tai QW, Tuxun T, Zhang JH, Zhao JM, Cao J, Muhetajiang $M$, et al. The role of laparoscopy in the management of liver hydatid cyst: a single-center experience and world review of the literature. Surg Laparosc Endosc Percutan Tech 2013;23:171-5. [CrossRef]

14. Bostanci O, Kartal K, Yazici P, Karabay O, Battal M, Mihmanli M. Laparoscopic versus open surgery for hydatid disease of the liver. A single center experience. Ann Ital Chir 2016;87:237-41.

15. Tuxun T, Zhang JH, Zhao JM, Tai QW, Abudurexti M, Ma HZ, et al. World review of laparoscopic treatment of liver cystic echinococcosis-914 patients. Int J Infect Dis 2014;24:43-50.

16. Jabbari Nooghabi A, Mehrabi Bahar M, Asadi M, Jabbari Nooghabi M, Jangjoo A. Evaluation and Comparison of the Early Outcomes of Open and Laparoscopic Surgery of Liver Hydatid Cyst. Surg Laparosc Endosc Percutan Tech 2015;25:403-7. [CrossRef]

17. He YB, Yao G, Tuxun T, Bai L, Li T, Zhao JM, et al. Efficacy of radical and conservative surgery for hepatic cystic echinococcosis: a meta-analysis. Int J Clin Exp Med 2015;8:7039-48. 